\title{
Foreword: Special Issue on Parameterized and Exact Computation
}

\author{
Jiong Guo ${ }^{1}$ Danny Hermelin ${ }^{1}$ \\ Published online: 28 November 2018 \\ (c) Springer Science+Business Media, LLC, part of Springer Nature 2018
}

We are pleased to present this special issue of Algorithmica, which contains seven extended journal papers selected from the twenty-eight papers presented at the 11th International Symposium on Parameterized and Exact Computation (IPEC) in Aarhus, Denmark. IPEC is a highly recognized annual meeting dedicated to publishing recent results in the fields of parameterized complexity and exact computation. The papers contained in this special issue went through a rigorous reviewing processing, according to the high standard of the journal.

In "Subexponential-time Algorithm for Maximum Independent Set in Pt-free and Broom-free Graphs," Gábor Bascó, Daniel Lokshtanov, Dániel Marx, Marcin Pilipczuk, Zsolt Tuza, and Erik Jan van Leeuwen prove that there exists an algorithm for the Maximum Independent Set on Pt-free graphs with a running time subexponentially depending on the number of vertices. The classic complexity of the problem is still open.

Serge Gaspers, Joachim Gudmundsson, Mitchell Jones, Julián Mestre, Stefan Rümmele in "Turbocharging Treewidth Heuristics" propose a parameterized algorithm for improving the heuristics for computing treewidth, that are based on computing elimination order. Experimental studies are also considered.

In "Backdoors for Linear Temporal Logic," Arne Meier, Sebastian Ordyniak, M. S. Ramanujan, and Irena Schindler study the parameterized complexity of the satisfiability problem parameterized by the size of backdoor and present some complexity classification results.

In "Clifford Algebras Meet Tree Decompositions," Michał Włodarczyk gives improved algorithm counting Steiner trees and Hamiltonian cycles, both parameterized by treewidth, whereby a new type of subset convolution has been introduced and Clifford algebra is used for computing the convolution.

Kitty Meeks investigates in "Randomised Enumeration of Small Witnesses Using a Decision Oracle" the complexity relation between the decision and enumeration

\footnotetext{
$凶$ Jiong Guo

jguo@sdu.edu.cn

1 Jinan, China
} 
versions of combinatorial problems, which rely on determining where there exists a subset of k elements with some specified property.

In "Fine-grained Dichotomies for the Tutte Plane and Boolean \#CSP," Cornelius Brand, Holger Dell, and Marc Roth complete the dichotomies for the compleixty of evaluting the Tutte polynomial at fixed points and for counting the number of satisfying assignments to a constraint satisfication instance over the Boolean domain.

Archontia C. Giannopoulou, Michał Włodarczyk, Jean-Florent Raymond, Dimitrios M. Thilikos, and Marcin Wrochna achieve two results concerning with the cutwidth, a size bound on the minimal immersion obstruction for cutwidth at most $\mathrm{k}$, and a new parameterized algorithm for computing cutwidth.

The editors would like to thank all authors and reviewers for contributing to this special issue. We would also like to express our appreciation to the editorial staff of Algorithmica for their assistance.

Publisher's Note Springer Nature remains neutral with regard to jurisdictional claims in published maps and institutional affiliations. 